\title{
Experimental demonstration of a 3D-printed nonlinear tuned vibration absorber
}

\author{
C. Grappasonni, G. Habib, T. Detroux G. Kerschen \\ Space Structures and Systems Laboratory (S3L) \\ Department of Aerospace and Mechanical Engineering \\ University of Liège, Liège, Belgium \\ chiara.grappasonni, giuseppe.habib, tdetroux, g.kerschen@ulg.ac.be
}

\begin{abstract}
Engineering structures are designed to be lighter and more flexible, hence reducing the extent of application of linear dynamic models. Concurrently, vibration mitigation is required for enhancing the performance, comfort or safety in real-life applications. Passive linear vibration absorbers are purpose-built, often designed using Den Hartog's equal-peak strategy. However, nonlinear systems are known to exhibit frequency-energy-dependent oscillations which linear absorbers cannot effectively damp out. In this context, the paper introduces a new nonlinear tuned vibration absorber (NLTVA) whose nonlinear functional form is tailored according to the frequency-energy dependence of the nonlinear primary structure. The NLTVA design aims at ensuring equal peaks in the nonlinear receptance function for an as large as possible range of forcing amplitudes, hence generalizing Den Hartog's method to nonlinear systems. Our focus in this study is on experimental demonstration of the NLTVA performance using a primary structure consisting of a cantilever beam with a geometrically nonlinear component at its free end. The absorber is implemented using a doubly-clamped beam fabricated thanks to 3D printing. The NLTVA performance is also compared with that of the classical linear tuned vibration absorber.
\end{abstract}

Keywords: Nonlinear resonances; tuned vibration absorber; equal-peak method; 3D printing; experimental demonstration.

\section{INTRODUCTION}

Engineering structures must often operate in a harsh dynamic environment. Passive vibration absorbers have therefore been exploited to mitigate the resonant vibrations with a limited increase in weight. Linear tuned vibration absorbers (LTVA) are commonly designed using the well-established Den Hartog's equal-peak strategy [1]. However, in view of its narrow bandwidth, the LTVA can be ineffective when the primary structure exhibits frequency-energy-dependent nonlinear oscillations. In this context, a nonlinear counterpart to the classical LTVA would be extremely beneficial, and several nonlinear absorbers have been proposed in the literature, see, e.g., [2-5].

The concept of a nonlinear tuned vibration absorber (NLTVA) was introduced by the authors in [6]. One unconventional aspect of the NLTVA is that the mathematical form of its restoring force is tailored according to the nonlinear restoring force of the primary system. The NLTVA parameters are then determined using a nonlinear generalization of Den Hartog's equal-peak method. In the present study, the NLTVA effectiveness is verified experimentally on a primary structure consisting of a cantilever beam with a geometrically nonlinear component at its free end.

The procedure that we propose herein to design and validate the NLTVA is sketched in Figure 1. Starting from an experimentallycharacterized structure, an equivalent nonlinear single-degree-of-freedom (SDOF) modal model of the targeted resonance is first 
derived (Section 2). From the nonlinear modal model, the analytic tuning procedure developed in [6] provides the absorber's parameters, and, in particular, its load-deflection characteristic (Section 3). The NLTVA is then implemented using doublyclamped geometrically nonlinear beams which are fabricated using 3D printers. The mechanical and geometrical properties of the nonlinear beams are also obtained analytically (Section 4). Finally, the NLTVA performance is verified experimentally through sine sweep testing at different excitation levels (Section 5).

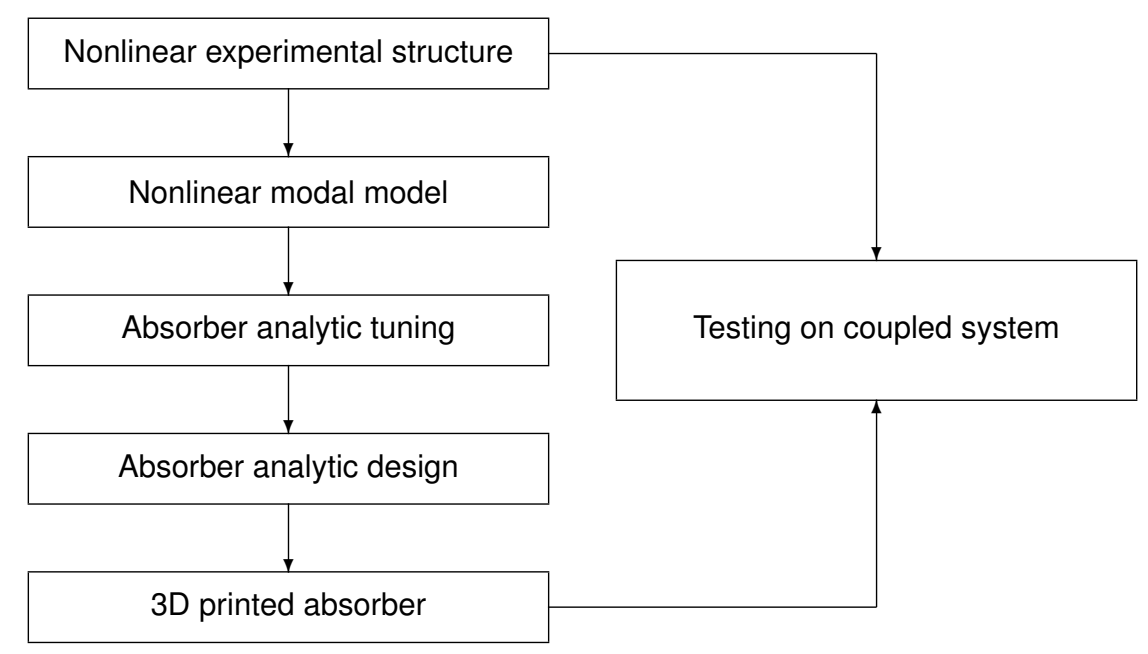

Figure 1: Proposed procedure for NLTVA design and performance validation.

\section{PRIMARY SYSTEM DESCRIPTION}

The primary system considered throughout this work is a benchmark that was originally proposed in [7] and that was studied by a number of research groups across the world. The set-up, shown in Figure 2, is a monolithic structure made of $42 \mathrm{CrMo} 4$ steel that comprises a cantilever beam with a very thin beam at its tip. The thin beam can exhibit large-displacement-related effects (i.e., mid-plane stretching effects) and behaves as a hardening nonlinearity. Around the first resonance, the system can be modeled as a Duffing oscillator.

The structure was instrumented with 7 accelerometers which span the beam regularly. A shaker located $0.3 \mathrm{~m}$ far from the clamped root was used to apply the excitation measured through a load cell. The signal acquisition was performed using the LMS SCADAS mobile and the LMS Test.LAB Structures Acquisition programs. The frequency-domain nonlinear subspace identification (FNSI) method [8] was used to determine the coefficient of the cubic spring modelling the nonlinear effect of the thin beam. The value that was identified from high-level random data with a $16 \mathrm{Nrms}$ forcing amplitude was $2.37 \times 10^{9} \mathrm{~N} / \mathrm{m}^{3}$. In addition, the FNSI method provided the modal parameters of the underling linear system listed in Table 1.

\begin{tabular}{ccc}
\hline Mode & Natural frequency $(\mathrm{Hz})$ & Damping ratio (\%) \\
1 & 31.05 & 0.12 \\
2 & 145.68 & 0.05 \\
\hline
\end{tabular}

TABLE 1: Modal parameters of the first two modes of the underlying linear structure.

The procedure for the tuning of the absorbers in [6] relies on the assumption of a SDOF primary system, which holds here, because the nonlinear beam has well-separated modes (Table 1). The mitigation of the resonance of the first beam mode is targeted. The frequency bandwidth of interest is therefore limited to [10-50] $\mathrm{Hz}$. The physical mass of the beam $m_{\text {beam }}$ is 


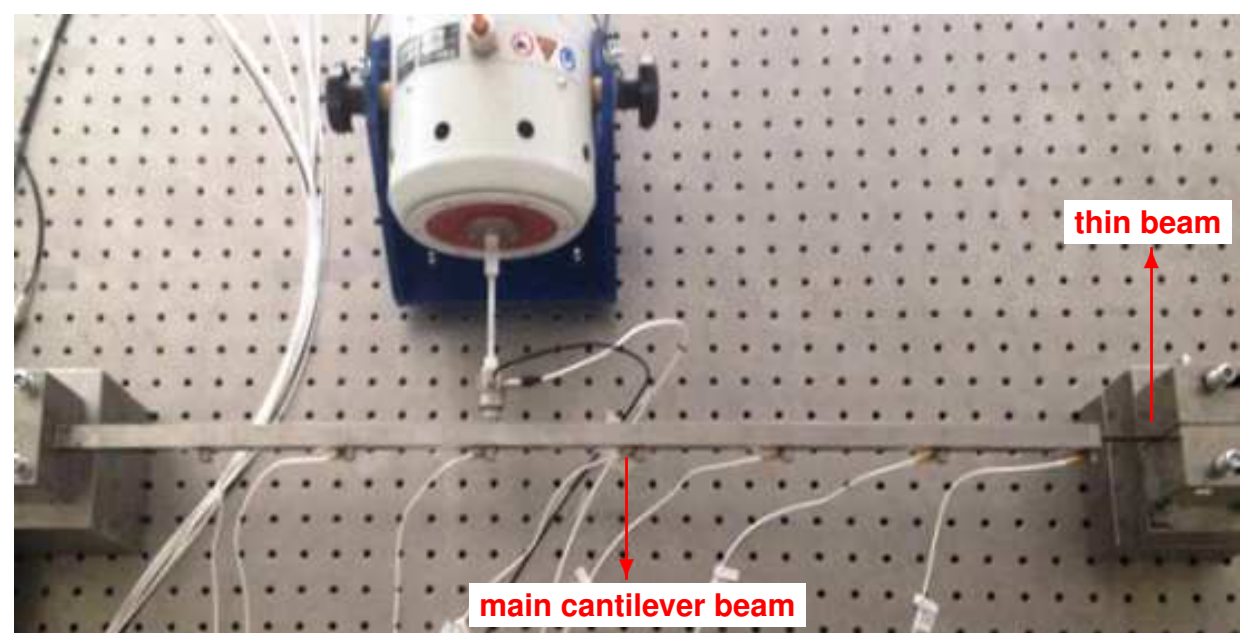

Figure 2: Experimental setup of the nonlinear beam.

$1.105 \mathrm{~kg}$. The effective mass $m_{1}$, damping $c_{1}$ and stiffness $k_{1}$ of the first mode can be calculated with respect to the degree of freedom to which the nonlinearity is attached. As depicted in Figure 4, unlike the LTVA which is realized using a cantilever beam, the NLTVA is implemented using a doubly-clamped beam that needs a support to be mounted on the primary structure. This support of mass $m_{\text {supp }}=0.14 \mathrm{~kg}$ is therefore part of the primary system for the coupled beam and NLTVA system. The effective mass of the first mode increases by a factor of $40 \%$, and the first natural frequency decreases by about $21 \%$. Table 2 summarizes the parameters of the nonlinear SDOF model of the beam around its first resonance, which can be seen as a Duffing oscillator.

\begin{tabular}{lcccc}
\hline Configuration & $m_{1}$ & $c_{1}$ & $k_{1}$ & $k_{n l 1}$ \\
w/o support (LTVA) & $0.2893 \mathrm{~kg}$ & $0.1357 \mathrm{Ns} / \mathrm{m}$ & $1.1009 \times 10^{4} \mathrm{~N} / \mathrm{m}$ & $2.3703 \times 10^{9} \mathrm{~N} / \mathrm{m}^{3}$ \\
w/ support (NLTVA) & $0.4090 \mathrm{~kg}$ & $0.2509 \mathrm{Ns} / \mathrm{m}$ & $1.0660 \times 10^{4} \mathrm{~N} / \mathrm{m}$ & $2.3703 \times 10^{9} \mathrm{~N} / \mathrm{m}^{3}$ \\
\hline
\end{tabular}

TABLE 2: SDOF models of the nonlinear beam for the configurations with LTVA/NLTVA.

\section{LINEAR AND NONLINEAR DEN HARTOG'S EQUAL-PEAK METHODS}

\subsection{The linear tuned vibration absorber (LTVA)}

The steady-state response of an undamped SDOF system subjected to a harmonic excitation at a constant frequency can be suppressed using an undamped linear tuned vibration absorber, as proposed by Frahm in 1909 [9]. However, the LTVA performance deteriorates significantly when the excitation frequency varies. To improve the performance robustness, damping was introduced in the absorber by Ormondroyd and Den Hartog [10]. The equations of motion of the coupled system are

$$
\begin{aligned}
m_{1} \ddot{x}_{1}+k_{1} x_{1}+c_{2}\left(\dot{x}_{1}-\dot{x}_{2}\right)+k_{2}\left(x_{1}-x_{2}\right) & =F \cos \omega t \\
m_{2} \ddot{x}_{2}+c_{2}\left(\dot{x}_{2}-\dot{x}_{1}\right)+k_{2}\left(x_{2}-x_{1}\right) & =0
\end{aligned}
$$

where $x_{1}(t)$ and $x_{2}(t)$ are the displacements of the harmonically-forced primary system and of the damped LTVA, respectively. Den Hartog proposed two approximate formulas for choosing the stiffness and damping ratio of the absorber in order to minimize the maximal value of the receptance function. In practice, the minimization corresponds to force the two resonant peaks to have the same height. Interestingly, it is only recently that the exact solution to this problem was found [11]. The introduction of linear damping into the primary system complicates further the problem. A series solution for minimizing the maximal frequency 
response was proposed in [12] and writes

$$
\begin{aligned}
m_{2}= & \epsilon m_{1} \\
k_{2}= & \epsilon k_{1}\left[\frac{1}{1+\epsilon}-\mu_{1} \frac{1}{1+\epsilon} \sqrt{\frac{1}{2(1+\epsilon)}\left(3+4 \epsilon-\frac{A B}{2+\epsilon}\right)}+\mu_{1}^{2} \frac{C_{0}-4(5+2 \epsilon) A B}{4(1+\epsilon)^{2}(2+\epsilon)(9+4 \epsilon)}\right]^{2} \\
c_{2}= & 2 \sqrt{k_{2} m_{2}}\left[\sqrt{\frac{3 \epsilon}{8(1+\epsilon)}}+\mu_{1} \frac{60+63 \epsilon+16 \epsilon^{2}-2(3+2 \epsilon) A B}{8(1+\epsilon)(2+\epsilon)(9+4 \epsilon)}+\right. \\
& \left.\mu_{1}^{2} \frac{C_{1}(A+B) \sqrt{2+\epsilon}+C_{2}(A-B) \sqrt{\epsilon}}{32(1+\epsilon)(2+\epsilon)^{2}(9+4 \epsilon)^{3} \sqrt{2 \epsilon(1+\epsilon)}}\right]
\end{aligned}
$$

where

$$
\begin{aligned}
\mu_{1} & =\frac{c_{1}}{2 \sqrt{m_{1} k_{1}}} \\
A & =\sqrt{3(2+\epsilon)-\sqrt{\epsilon(2+\epsilon)}} \\
B & =\sqrt{3(2+\epsilon)+\sqrt{\epsilon(2+\epsilon)}} \\
C_{0} & =52+41 \epsilon+8 \epsilon^{2} \\
C_{1} & =-1296+2124 \epsilon+6509 \epsilon^{2}+5024 \epsilon^{3}+1616 \epsilon^{4}+192 \epsilon^{5} \\
C_{2} & =48168+112887 \epsilon+105907 \epsilon^{2}+49664 \epsilon^{3}+11632 \epsilon^{4}+1088 \epsilon^{5}
\end{aligned}
$$

and $\epsilon=m_{2} / m_{1}$ is the mass ratio. We note that Eqs. (2) reduce to Den Hartog's approximate formulas[13] for undamped main system $\left(\mu_{1}=0\right)$ :

$$
\begin{aligned}
& k_{2}=\epsilon k_{1}\left[\frac{1}{1+\epsilon}\right]^{2} \\
& c_{2}=2 \sqrt{k_{2} m_{2}} \sqrt{\frac{3 \epsilon}{8(1+\epsilon)}}
\end{aligned}
$$

Considering the harmonically-forced, lightly-damped Duffing oscillator derived in Section 2, the performance of the LTVA attached to this nonlinear system is investigated. The equations of motion of the coupled system are

$$
\begin{aligned}
m_{1} \ddot{x}_{1}+c_{1} \dot{x}_{1}+k_{1} x_{1}+k_{n l 1} x_{1}^{3}+c_{2}\left(\dot{x}_{1}-\dot{x}_{2}\right)+k_{2}\left(x_{1}-x_{2}\right) & =F \cos \omega t \\
m_{2} \ddot{x}_{2}+c_{2}\left(\dot{x}_{2}-\dot{x}_{1}\right)+k_{2}\left(x_{2}-x_{1}\right) & =0 .
\end{aligned}
$$

The tuning of the LTVA is performed considering a physical mass ratio, $\epsilon_{\text {phys }}=m_{2} /\left(m_{\text {beam }}\right)$, of $3 \%$. This constraint directly sets the LTVA mass to $3.315 \times 10^{-2} \mathrm{~kg}$, and, as a consequence, the effective mass ratio is $\epsilon=m_{2} / m_{1}=11.46 \%$. The parameters of the optimal LTVA, calculated from Eqs. (2) with the values given in Table 2, are listed in Table 3.

Figure 3 (a) shows the ratio between the displacement response at beam tip and the applied force for various forcing amplitudes, namely $F=1.0 \mathrm{~N}, F=3.5 \mathrm{~N}, F=6.5 \mathrm{~N}$ and $F=9.5 \mathrm{~N}$. The frequency response curves are computed using a path-following algorithm combining shooting and pseudo-arclength continuation [14]. For low values of forcing amplitude, the two resonant peaks have similar amplitude, which validates the effectiveness of the equal-peak rule for linear regimes of motions. However, increasing the forcing amplitude activates the nonlinearity of the thin beam. As a result, the amplitude of the second resonant peak increases significantly, which confirms that the LTVA is ineffective in nonlinear regimes of motion.

\subsection{The nonlinear tuned vibration absorber (NLTVA)}

In [6], following previous studies [15,16], a nonlinear vibration absorber, termed the nonlinear tuned vibration absorber (NLTVA), was proposed. One unconventional feature of this absorber is that the mathematical form of the restoring force is not determined a priori, but it is chosen as a "mirror" of the primary system's restoring force. In other words, if the primary system presents linear and cubic springs, the NLTVA should also possess linear and cubic springs. The absorber parameters are then determined so 
as to ensure equal peaks in nonlinear regimes of motion, giving rise to an effective reduction of the resonance response in a large range of forcing amplitudes.

The equations of motion of the Duffing oscillator of Section 2 with an attached NLTVA are

$$
\begin{aligned}
m_{1} \ddot{x}_{1}+c_{1} \dot{x}_{1}+k_{1} x_{1}+k_{n l 1} x_{1}^{3}+c_{2}\left(\dot{x}_{1}-\dot{x}_{2}\right)+k_{2}\left(x_{1}-x_{2}\right)+k_{n l 2}\left(x_{1}-x_{2}\right)^{3} & =F \cos \omega t \\
m_{2} \ddot{x}_{2}+c_{2}\left(\dot{x}_{2}-\dot{x}_{1}\right)+k_{2}\left(x_{2}-x_{1}\right)+k_{n l 2}\left(x_{2}-x_{1}\right)^{3} & =0
\end{aligned}
$$

The tuning rules for the linear parameters of the NLTVA, $k_{2}$ and $c_{2}$, are those presented in Eqs.(2). For the nonlinear coefficient $k_{n l 2}$, it is provided in reference [6]:

$$
k_{n l 2}=\frac{2 \epsilon^{2}}{(1+4 \epsilon)} k_{n l 1}
$$

An interesting observation is that the ratio between the two nonlinear stiffnesses is linear and depends only on the mass ratio $\epsilon$, a quite surprising finding for a nonlinear system. As for the LTVA, a physical mass ratio, $\epsilon_{\text {phys }}=m_{2} /\left(m_{\text {beam }}+m_{\text {supp }}\right)$, of $3 \%$ is considered. Hence, the NLTVA mass is $3.735 \times 10^{-2} \mathrm{~kg}$, and the effective mass ratio is $\epsilon=m_{2} / m_{1}=9.13 \%$. The NLTVA parameters can be calculated from Eqs. $(2,7)$ and Table 2; they are listed in Table 3.

\begin{tabular}{lcccc}
\hline & $m_{2}$ & $c_{2}$ & $k_{2}$ & $k_{n l 2}$ \\
LTVA & $3.315 \times 10^{-2} \mathrm{~kg}$ & $2.279 \mathrm{Ns} / \mathrm{m}$ & $1.014 \times 10^{3} \mathrm{~N} / \mathrm{m}$ & - \\
NLTVA & $3.735 \times 10^{-2} \mathrm{~kg}$ & $1.959 \mathrm{Ns} / \mathrm{m}$ & $8.161 \times 10^{2} \mathrm{~N} / \mathrm{m}$ & $2.896 \times 10^{7} \mathrm{~N} / \mathrm{m}^{3}$ \\
\hline
\end{tabular}

TABLE 3: SDOF model of the LTVA and NLTVA.

The effectiveness of the NLTVA is proved in Figure $3 \mathrm{~b}$ for the same primary system already considered for the LTVA. It can be noted that the strong detuning characterizing the LTVA does no longer occur. When the forcing amplitude $F$ increases, the peaks of the frequency response function $x_{1} / F$ remain equal or almost equal for the largest forcing amplitude.

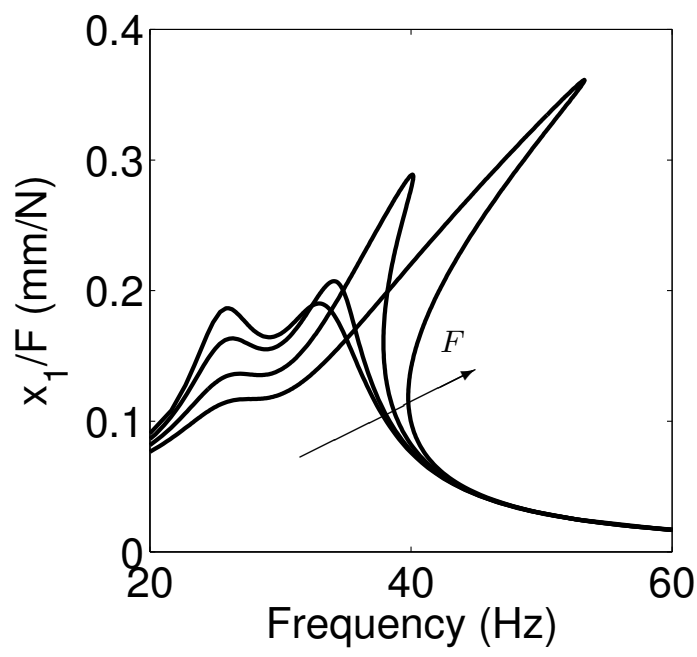

(a) LTVA

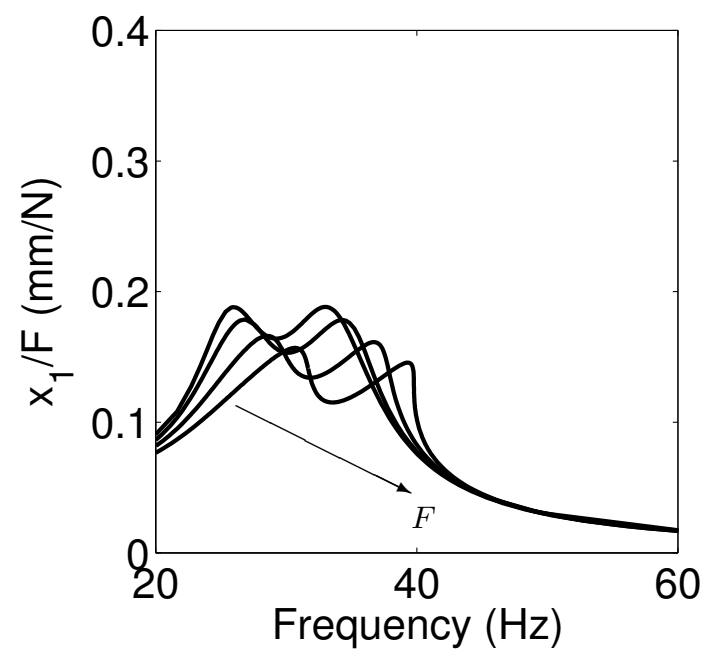

(b) NLTVA

Figure 3: Ratio between the frequency response and the force in a Duffing oscillator with the LTVA or the NLTVA attached. For the different curves the forcing amplitude is $F=1.0 \mathrm{~N}, F=3.5 \mathrm{~N}, F=6.5 \mathrm{~N}$ and $F=9.5 \mathrm{~N}$. 


\section{PRACTICAL REALIZATION OF LTVA AND NLTVA USING CANTILEVER AND DOUBLY-CLAMPED BEAMS}

Different beam models are used for the LTVA and NLTVA, since the stiffness requirements for the tuning are not the same. Specifically, linear cantilever beams are considered for the practical implementation of the LTVA, whereas nonlinear doublyclamped beams are considered for the NLTVA. Both absorbers were manufactured using a Statasys 3D printer with the Polyjet Digital ABS material. This material is specifically designed to simulate engineering plastics with high strength, and it also offers the possibility to create thin-walled parts. The mechanical properties are given in Table 4.

\begin{tabular}{cccc}
\hline Young Modulus & Density & Elongation at break & minimum thickness \\
$1950 \mathrm{MPa}$ & $1175 \mathrm{~kg} / \mathrm{m}^{3}$ & $32 \%$ & $0.5 \mathrm{~mm}$ \\
\hline
\end{tabular}

TABLE 4: Polyjet Digital ABS material properties.

\subsection{LTVA cantilever beam}

The design of a linear cantilever beam of mass $m_{2}$ and of first natural frequency $\omega_{2}=\sqrt{k_{2} / m_{2}}$ is a classical problem that, solved with respect to the beam length $L$, yields

$$
L=\left(\frac{1.875^{4} E m_{2}}{12 \omega_{2}^{2} \rho^{2}}\right)^{1 / 5}
$$

where $E$ is the material's Young modulus and $\rho$ is the material's density. To guarantee that the beam behaves linearly, large deflections have to be avoided for the operating loading conditions. Therefore, a squared cross section of thickness $t$ and width $w$

$$
t=w=\left(\frac{m_{2}}{\rho L}\right)^{1 / 2}
$$

is utilized. The geometrical dimensions of the cantilever beam are obtained from the parameters in Table 3 and are listed in Table 5.

\subsection{NLTVA doubly-clamped beam}

According to the mirror rule, the NLTVA should behave as a Duffing oscillator around the resonance of interest. Doubly-clamped beams are suitable candidates for NLTVA realization, because the mid-plane stretching effect, not present in cantilever beams, can quickly activate nonlinear behaviors. Another advantage is that the geometrical dimensions of uniform doubly-clamped beams can be derived analytically as functions of only the linear $k_{2}$ and nonlinear $k_{n l 2}$ stiffness coefficients in Eqs. (2) and (7), respectively:

$$
k_{2}=2 \pi^{4} \frac{E I}{L^{3}}, \quad k_{n l 2}=\pi^{4} \frac{E A}{8 L^{3}}
$$

in which $A$ and $I$ are the cross-section area and momentum of inertia, respectively. The analytic derivation is explained in [17].

It is interesting to observe that the coefficient of the nonlinear term depends on the axial stiffness of the beam $E A$, as in [18]. Therefore, the cross-section dimensions are the geometrical parameters driving the design. In fact, the ratio between the nonlinear and linear coefficients depends only on the area and the momentum of inertia. For rectangular cross sections, the thickness $t$ can be directly deduced from this relationship

$$
t=\sqrt{\frac{3 k_{2}}{4 k_{n l 2}}}
$$

whereas the beam length $L$ and the cross-section width $w$ can be chosen to satisfy the following constraint

$$
\frac{L^{3}}{w}=\frac{\pi^{4} E t}{8 k_{n l 2}}
$$


Once the material is chosen, the design of a rectangular doubly-clamped beam capable to follow a prescribed cubic loaddeflection curve reduces to a problem that admits multiple solutions. An additional constraint can therefore still be imposed (e.g., manufacturing limitations). The linear and nonlinear stiffness coefficients in Table 3 are used to evaluate the geometrical dimensions of the doubly-clamped beam from Eqs. (11-12), and the results are given in Table 5. We remark that the mass of the designed doubly-clamped beam is much lower than the value assigned to $m_{2}$ in Table 3; a lumped mass is then added at midspan so that the nonlinear beam behaves as a SDOF NLTVA. We also note that there was no attempt at tuning the NLTVA damping through, e.g., the selection of a proper material.

\begin{tabular}{|c|c|c|c|c|}
\hline Beam & Length & Width & Thickness & Mass \\
\hline cantilever (LTVA) & $263.0 \mathrm{~mm}$ & $10.4 \mathrm{~mm}$ & $10.4 \mathrm{~mm}$ & $3.3 \times 10^{-2} \mathrm{~kg}$ \\
\hline doubly-clamped (NLTVA) & $236.5 \mathrm{~mm}$ & $4.5 \mathrm{~mm}$ & $4.4 \mathrm{~mm}$ & $0.6 \times 10^{-2} \mathrm{~kg}$ \\
\hline
\end{tabular}

TABLE 5: Geometrical dimensions of the cantilever and doubly-clamped beams.

\subsection{Experimental characterization of the manufactured absorbers}

The LTVA and NLTVA were manufactured using 3D-printing and are shown in Figure 4. Specifically, the doubly-clamped beam and its support were produced as a monolithic structure to ensure appropriate clamped-clamped boundary conditions.

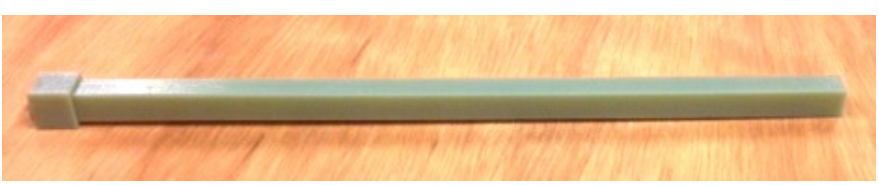

(a) LTVA

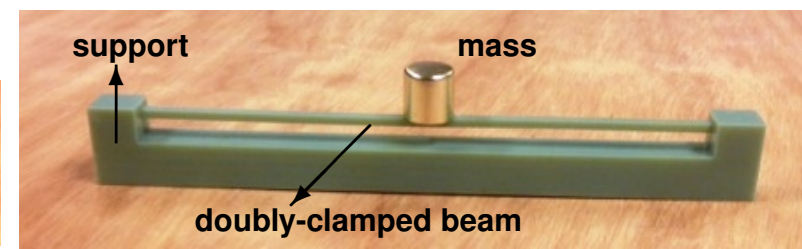

(b) NLTVA

Figure 4: LTVA and NLTVA manufactured using 3D-printing

In order to characterize the manufactured absorbers, the restoring force surface (RFS) method was employed since it yields a convenient visualization of the restoring force. For this purpose, the absorbers were mounted on the shaker, and a base excitation test was carried out using sine sweep around the first resonance. The accelerations at the base $\ddot{x}_{\text {base }}$ and at the point on the beam experiencing the maximum deflection (i.e., the tip of the cantilever beam and the midspan of the doubly-clamped beam) $\ddot{x}$ were measured and integrated once and twice to evaluate velocities and displacements, respectively. The RFS method relies on Newton's second law of motion, which can be written for the case under investigation as

$$
m \ddot{x}+f\left(x-x_{\text {base }}, \dot{x}-\dot{x}_{\text {base }}\right)=0
$$

where $f$ is the restoring force of the system. If the relative displacement is defined as $y=x-x_{b a s e}$, this equation recasts into

$$
f(y, \dot{y})=-m \ddot{x}
$$

A direct access to a nonparametric estimate of the restoring force surface defined by the triplets $(y, \dot{y},-m \ddot{x})$ is then obtained. The intersection of this surface with the zero-velocity plane provides the stiffness curve. Figure 5 represents the experimentallyevaluated stiffness curves for the LTVA and the NLTVA. There is a very close agreement between the measured and prescribed stiffness curves, both for the LTVA and NLTVA. Fitting with measured LTVA curves with a line provides an estimate of the linear stiffness equal to $1.037 \times 10^{3} \mathrm{~N}$, which is only in error of $2 \%$ with respect to the prescribed value. For the NLTVA, the fitting with a polynomial with linear and cubic terms provides the linear and cubic coefficients, i.e., $7.449 \times 10^{2} \mathrm{~N} / \mathrm{m}$ and $2.861 \times 10^{7} \mathrm{~N} / \mathrm{m}^{3}$, respectively. They correspond to an error of $9 \%$ and $1 \%$, respectively. Finally, we notice that the experimental dots in Figure 5 


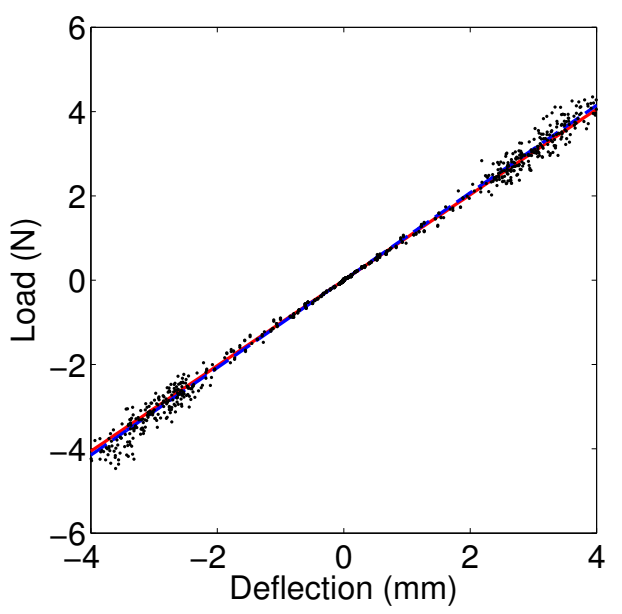

(a) LTVA

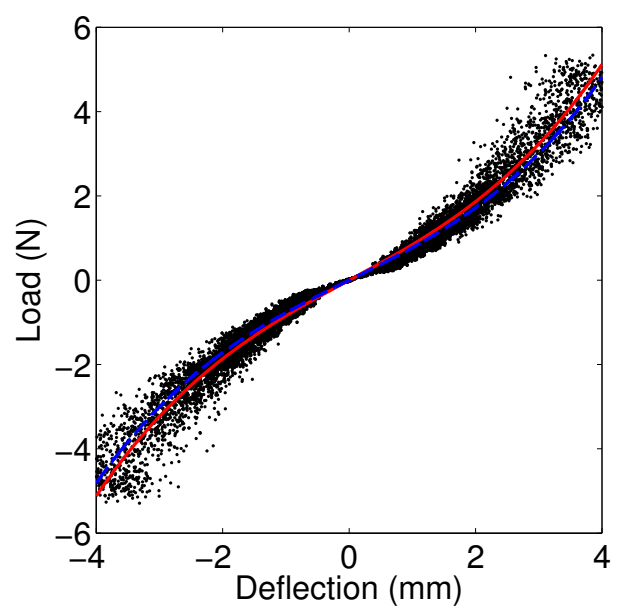

(b) NLTVA

Figure 5: Stiffness curves of the 3D-printed absorbers: prescribed (red lines), measured (black dots) and fitted (blue dashed lines) curves.

closely follow the prescribed curves for small deflections, whereas they are more scattered at the outer limits of the interval. This is attributed to the extrapolation of the points of the stiffness curve using the RFS method. Indeed, the points of the 3D surface with relative velocity practically equal to 0 are not in sufficient number to draw the stiffness curve, and this curve has to be traced by considering points with small, but non-zero, relative velocity. A dependency of the load on the relative velocity was also responsible for the scattering of the outer points.

\section{EXPERIMENTAL DEMONSTRATION OF LTVA AND NLTVA PERFORMANCE}

In this section the LTVA and NLTVA are attached to the nonlinear beam, as depicted in Figure 6 . The first objective is to confirm

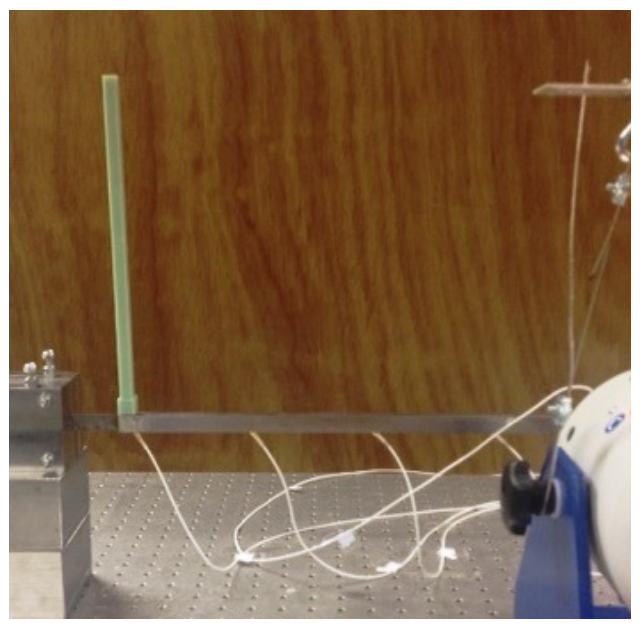

(a) LTVA

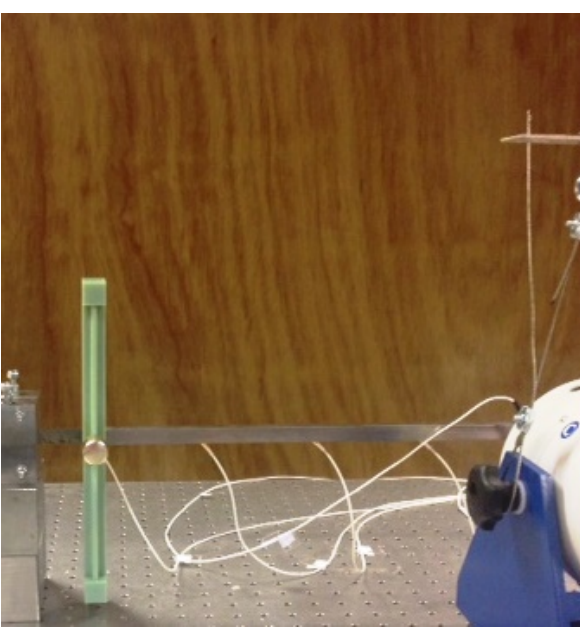

(b) NLTVA

Figure 6: Primary structure with attached LTVA and NLTVA. 
that the LTVA is effective only in linear regimes of motion, as discussed in Section 3.1. The second objective is to demonstrate that this limitation can be overcome by the NLTVA, as claimed in Section 3.2.

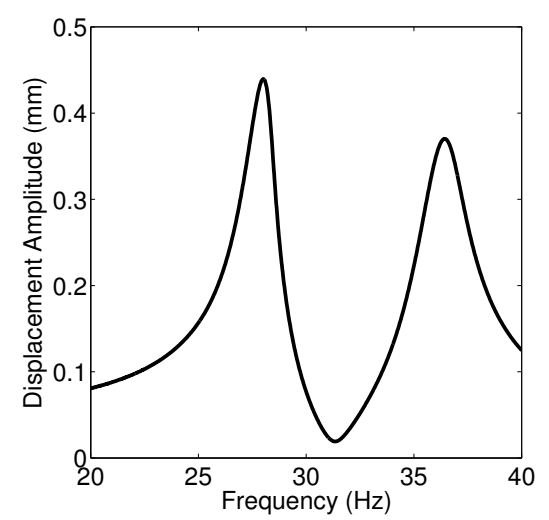

(a)

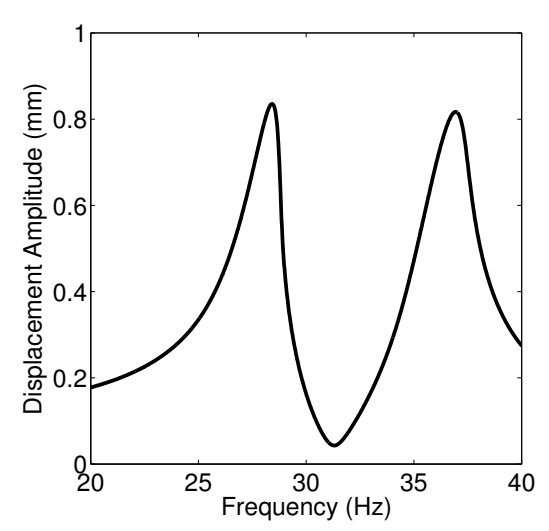

(d)

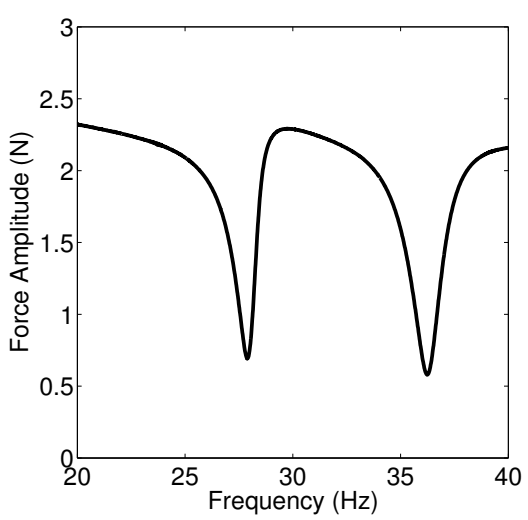

(b)

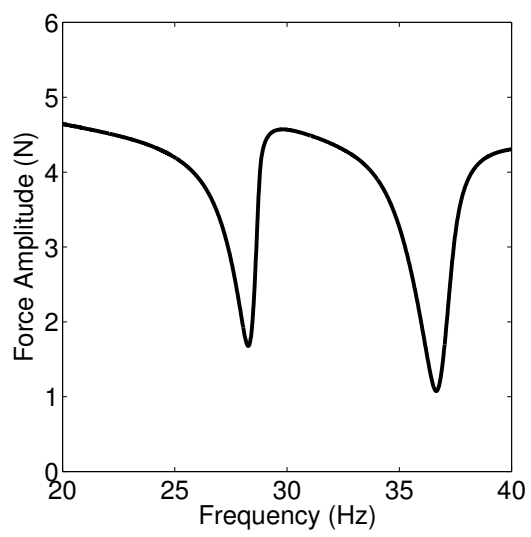

(e)

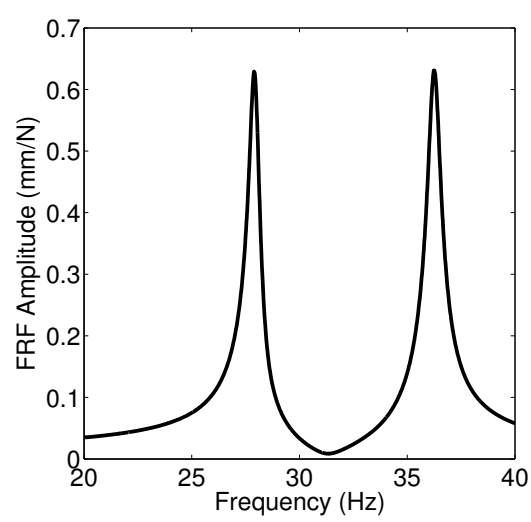

(c)

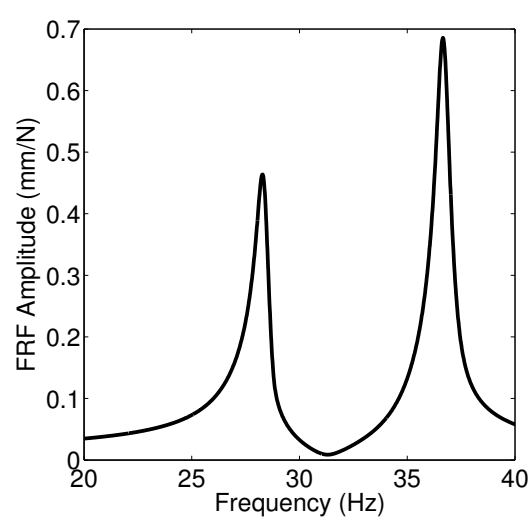

(f)

Figure 7: Experimental responses of the primary system with an attached LTVA (sine sweep excitation). (a,d) Displacement amplitude at the main beam tip, (b,e) force amplitude at the driving point, (c,f) FRF amplitude, for low and high excitation levels, respectively.

The coupled beam and LTVA system is first analyzed. Figure 7(a) plots the amplitude of the displacement at the main beam tip where the nonlinearity is located for sine sweep excitation at low level. Two resonance peaks of unequal amplitudes are observed. This discrepancy can be explained by analyzing the force signal measured at the interface between the structure and the shaker, represented in Figure 7(b). In fact, the experimental test is not performed at constant forcing level, although the voltage provided to the shaker is kept constant. Specifically, drops in the force amplitude can be noted at the resonant frequencies, a phenomenon that is typically encountered during vibration testing. However, the corresponding frequency response function (FRF) depicted in Figure 7(c) displays two equal peaks, which validates the LTVA design and the effectiveness of this absorber for linear regimes. When the driving signal provided to the shaker is doubled, the displacement amplitude increases as in Figure 7(d), and the resonance peaks seem to get closer. Nevertheless, the drops in the corresponding forcing amplitude are not equal, as shown in Figure 7(e). The FRF in Figure 7(f) presents two peaks of unequal amplitude, meaning that a detuning of the LTVA has happened.

Figures 8(a-c) depict the plots of the NLTVA performance for low-level sine sweep excitation. The FRF in Figure 8(c) shows two equal peaks, which can be considered as a proof of the NLTVA effectiveness when the system behaves linearly. The presence of the NLTVA support affects the primary structure's resonances, as already point out in Section 2. Therefore, the NLTVA curves are shifted toward lower frequencies in contrast to the LTVA. Nevertheless, this effect does not affect the findings of this paper, because the absorbers were tuned accordingly. Figures $8(d-f)$ presents the displacement, force and FRF amplitude, respectively, 


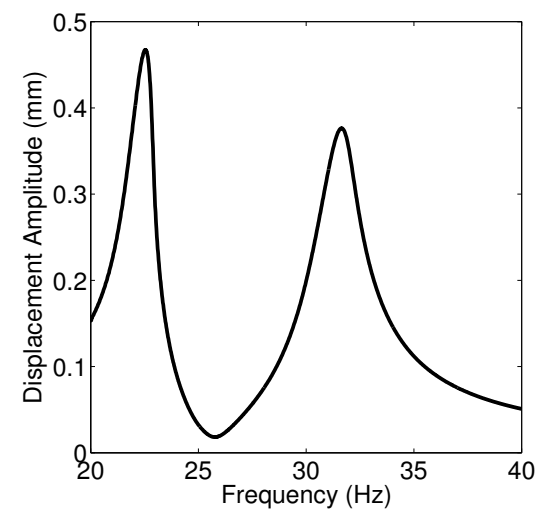

(a)

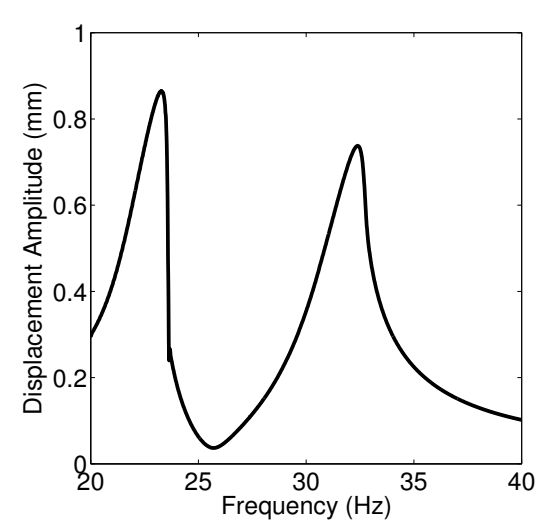

(d)

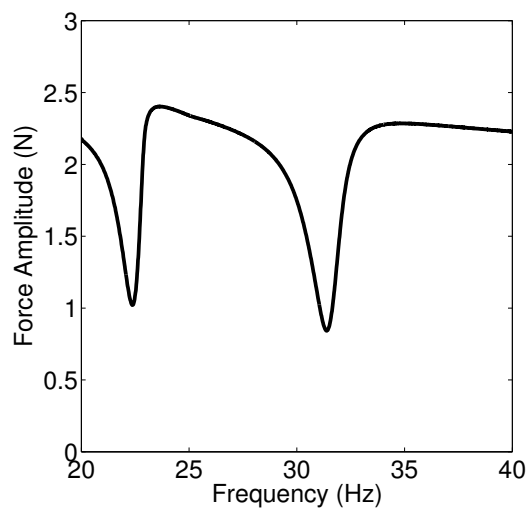

(b)

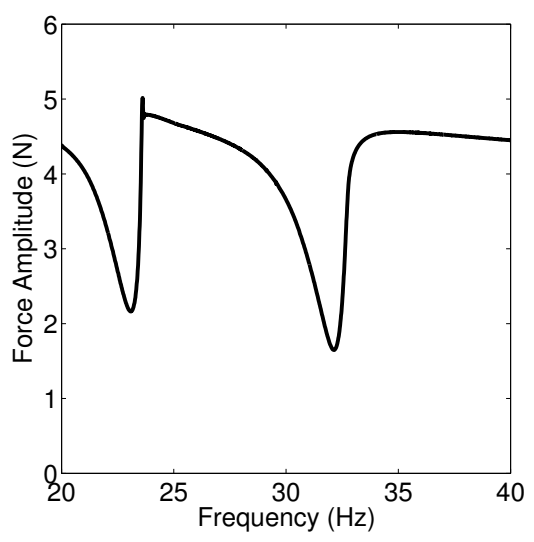

(e)

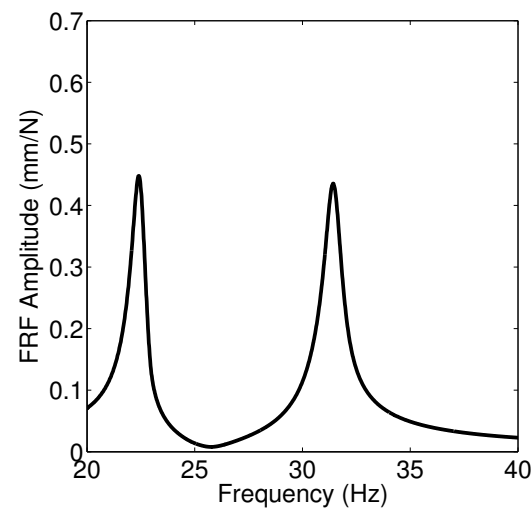

(c)

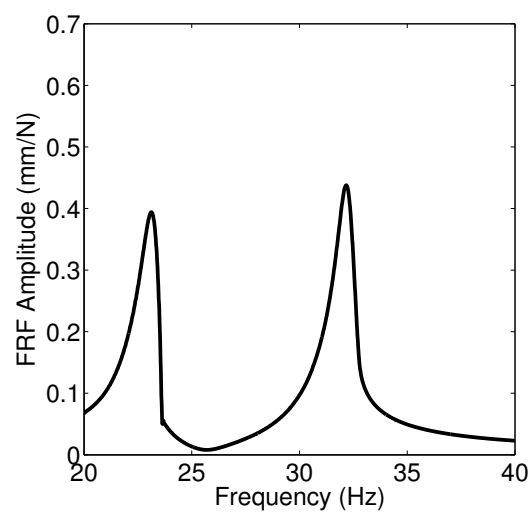

(f)

Figure 8: Experimental responses of the primary system with an attached NLTVA (sine sweep excitation). (a-d) Displacement amplitude at the main beam tip, (b-e) force amplitude at the driving point, (c-f) FRF amplitude, for low and high excitation levels, respectively.

when the driving signal provided to the shaker is doubled. The two resonance peaks in the FRF remains equal although the energy supplied to the system is high enough to activate the nonlinearity in the thin beam. Unlike the LTVA, the NLTVA is still effective at this forcing level.

In order to better quantify the detuning of the LTVA caused by the frequency-energy dependence of the oscillations, Figure 9(a) shows the displacement amplitude of the two resonant peaks at their corresponding force amplitudes for various excitation levels. The peak amplitudes are equal for low forcing amplitudes, but then they rapidly diverge from each other for forces greater than $1 \mathrm{~N}$. After this point the amplitude of one resonant peak increases significantly making the absorber ineffective. For the NLTVA, two resonant peaks with virtually the same amplitude are observed for all considered excitation levels in Figure 9(b), which is a confirmation of the excellent performance of the nonlinear absorber.

A final remark is that the coupled primary structure and NLTVA system seems to obey the superposition principle in Figure 9(b). Indeed, the response increases almost linearly with the forcing amplitude, whereas the coupled system vibrates in nonlinear regimes of motion. It therefore seems that the addition of a nonlinear attachment to a nonlinear system can somehow provides more linear dynamics. This interesting phenomenon will be investigated more closely in future studies. 


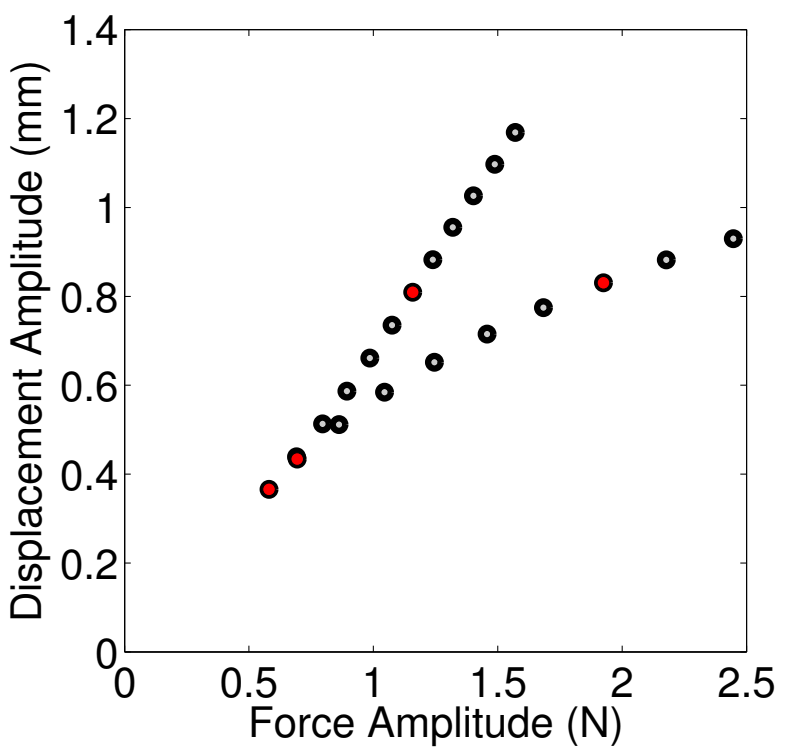

(a) LTVA

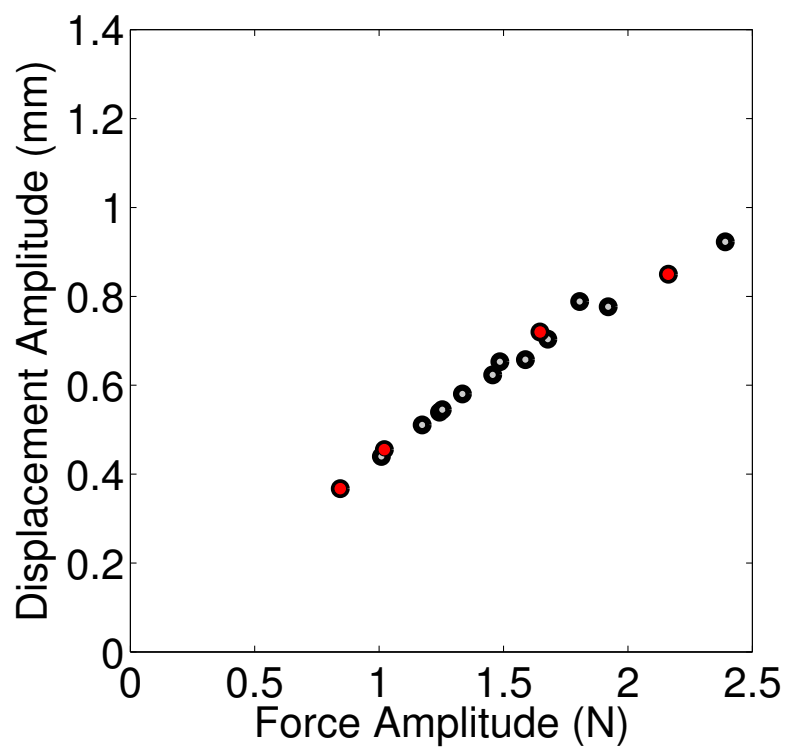

(b) NLTVA

Figure 9: Amplitude of the resonance peaks at the tip of the primary beam for increasing forcing amplitudes (LTVA and NLTVA). The red dots represent the values in Figures 7 and 8.

\section{CONCLUDING REMARKS}

The objective of this study was to verify experimentally the effectiveness of a new nonlinear tuned vibration absorber, the NLTVA. A complete procedure starting from the experimental identification of the primary structure to the testing of the coupled system was also proposed. An important feature of this methodology is that the nonlinear doubly-clamped beam implementing the NLTVA in practice and manufactured using 3D printing could be designed by resorting only to analytic formulas. The experimental test campaign demonstrated that the NLTVA clearly outperforms its linear counterpart (the LTVA), since this former absorber is able to maintain equal peaks in nonlinear regimes of motion.

\section{ACKNOWLEDGEMENTS}

The authors would like to ackonwledge the financial support of the European Union (ERC Starting Grant NoVib 307265). The authors also want to thank LMS A Siemens Business for providing access to the LMS Test.Lab software.

\section{REFERENCES}

[1] Den Hartog, J. P., Mechanical Vibrations, McGraw-Hill, New York, 1934.

[2] Oueini, S. and Nayfeh, A., Analysis and Application of a Nonlinear Vibration Absorber, Journal of Vibration and Control, Vol. 6, pp. 999-1016, 2000.

[3] Vyas, A. and Bajaj, A., Dynamics of Autoparametric Vibration Absorbers using Multiple Pendulums, Journal of Sound and Vibration, Vol. 246, pp. 115-135, 2001.

[4] Vakakis, A. and Gendelman, O. V., Energy Pumping in Nonlinear Mechanical Oscillators, Part II: Resonance Capture, Journal of Applied Mechanics, Vol. 68, pp. 42-48, 2001.

[5] Vakakis, A. F., Gendelman, O., Bergman, L. A., McFarland, D. M., Kerschen, G. and Lee, Y. S., Nonlinear Targeted Energy Transfer in Mechanical and Structural Systems, Series: Solid Mechanics and Its Applications, Springer, 2009. 
[6] Habib, G., Detroux, T., Viguié, R. and Kerschen, G., Nonlinear generalization of Den Hartog's equal-peak method, Mechanical Systems and Signal Processing, 2014, in press.

[7] Thouverez, F., Presentation of the ECL benchmark, Mechanical Systems and Signal Processing, Vol. 17, No. 1, pp. 195 202, 2003.

[8] Noël, J. P. and Kerschen, G., Frequency-domain subspace identification for nonlinear mechanical systems, Mechanical Systems and Signal Processing, Vol. 40, pp. 701-717, 2013.

[9] Frahm, H., Device for Damping Vibrations of Bodies, US Patent No. 989958, 1909.

[10] Ormondroyd, J. and Den Hartog, J. P., The theory of the dynamic vibration absorber, Transaction of ASME, Vol. 50, pp. 9-22, 1928.

[11] Asami, T. and Nishihara, O., Closed-form Exact Solution to $\mathrm{H}_{\infty}$ Optimization of Dynamic Vibration Absorbers (Application to Different Transfer Functions and Damping Systems), Journal of Vibration and Acoustics, Vol. 125, pp. 398-405, 2003.

[12] Asami, T., Nishihara, O. and Baz, A. M., Analytical Solutions to $\mathrm{H}_{\infty}$ and $\mathrm{H}_{2}$ Optimization of Dynamic Vibration Absorbers Attached to Damped Linear Systems, Journal of Vibration and Acoustics, Vol. 124, pp. 284-295, 2002.

[13] Den Hartog, J. P., Mechanical Vibrations, McGraw-Hill, New York, 1985.

[14] Peeters, M., Viguié, R., Sérandour, G., Kerschen, G. and Golinval, J. C., Nonlinear Normal Modes, Part II: Toward a Practical Computation using Numerical Continuation, Mechanical Systems and Signal Processing, Vol. 23, pp. 195-216, 2009.

[15] Viguié, R. and Kerschen, G., On the functional form of a nonlinear vibration absorber, Journal of Sound and Vibration, Vol. 329, No. 25, pp. 5225-5232, 2010.

[16] Denman, H. H., Tautochronic bifilar pendulum torsion absorbers for reciprocating engines, Journal of Sound and Vibration, Vol. 159, No. 2, pp. 251-277, 1992.

[17] Grappasonni, C., Habib, G., Detroux, T., Wang, F., Kerschen, G. and Jensen, J., Practical design of a nonlinear tuned vibration absorber, Proceedings of the ISMA 2014 conference, 2014.

[18] Senturia, S., Microsystem design, Vol. 3, Kluwer academic publishers, Boston, 2001. 